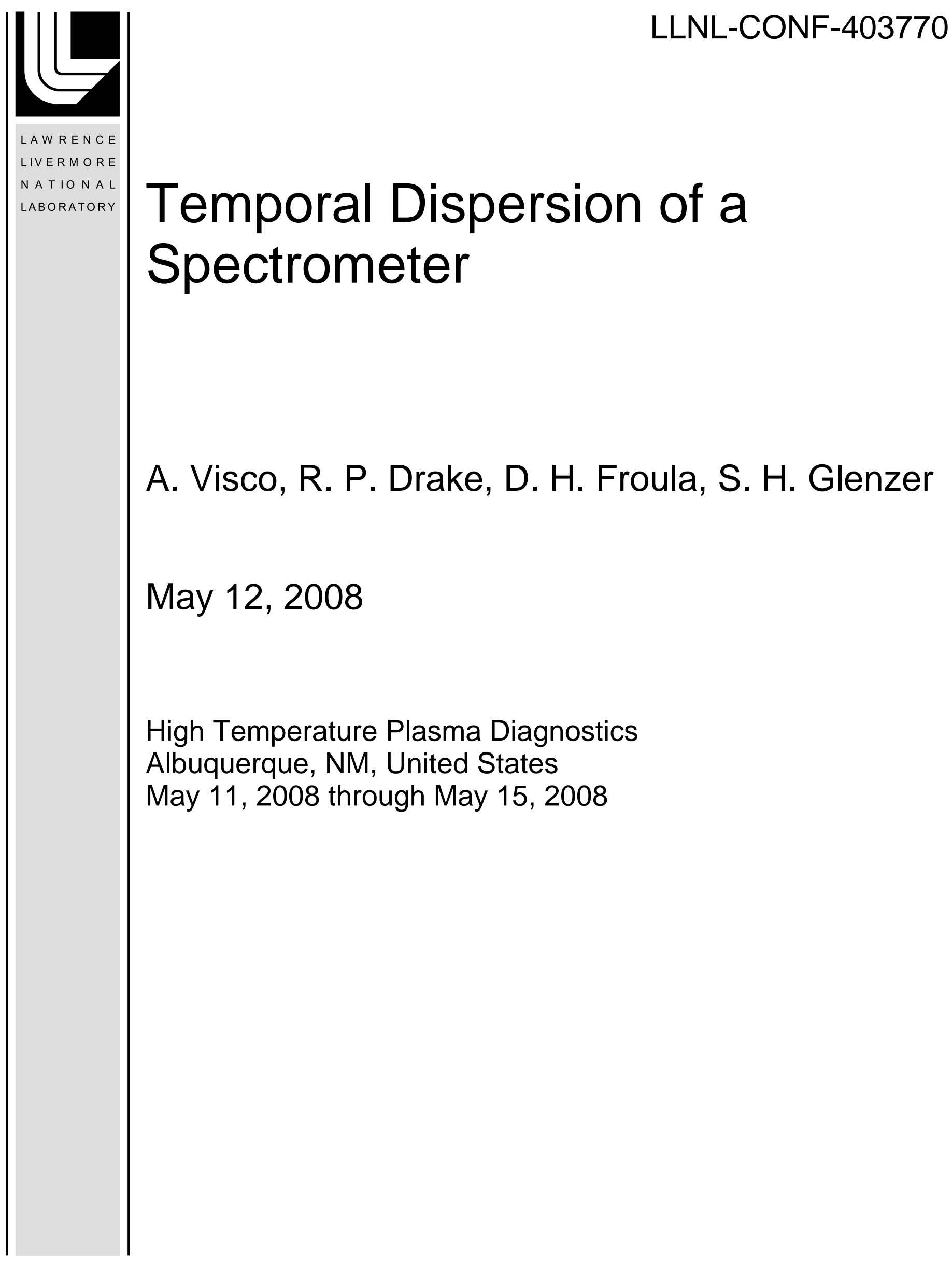


This document was prepared as an account of work sponsored by an agency of the United States government. Neither the United States government nor Lawrence Livermore National Security, LLC, nor any of their employees makes any warranty, expressed or implied, or assumes any legal liability or responsibility for the accuracy, completeness, or usefulness of any information, apparatus, product, or process disclosed, or represents that its use would not infringe privately owned rights. Reference herein to any specific commercial product, process, or service by trade name, trademark, manufacturer, or otherwise does not necessarily constitute or imply its endorsement, recommendation, or favoring by the United States government or Lawrence Livermore National Security, LLC. The views and opinions of authors expressed herein do not necessarily state or reflect those of the United States government or Lawrence Livermore National Security, LLC, and shall not be used for advertising or product endorsement purposes. 


\title{
Temporal Dispersion of a Spectrometer
}

\author{
A.Visco, R.P. Drake \\ University of Michigan, 2455 Hayward, Ann Arbor, MI, 48109 \\ D.H. Froula, S.H. Glenzer \\ L-399, Lawrence Livermore National Laboratory, P.O. Box 808, Livermore, CA 94551
}

The temporal dispersion of an optical spectrometer has been characterized for a variety of conditions related to optical diagnostics to be fielded at the National Ignition Facility (e.g., Full-Aperture Backscatter Station, Thomson Scattering). Significant time smear is introduced into these systems by the path length difference through the spectrometer. The temporal resolution can be calculated to depend only on the order of the grating, wavelength, and the number of grooves illuminated. To enhance the temporal dispersion, the spectral gratings can be masked limiting the number of grooves illuminated. Experiments have been conducted to verify these calculations. The size and shape of masks are investigated and correlated to the exact shape of the temporal instrument function, which is required when interpreting temporally resolved data. The experiments used a 300 fs laser pulse and a picosecond optical streak camera to determine the temporal dispersion. This was done for multiple spectral orders, gratings, and optical masks.

\section{INTRODUCTION}

Optical spectrometers are instruments employed by several diagnostics systems at many high energy density facilities, including the full-aperture backscattering station at the National Ignition Facility. Since plasmas produced by intense laser light are often short lived, with parameters changing on the time scale of tens to hundreds of picoseconds, temporal resolution of spectral features is important to investigating these plasmas. One limitation on the temporal resolution is the time smear introduced by the spectrometer. The optical path length through the spectrometer varies for rays reflected from different parts of the grating, causing a delay for different parts of the pulse. The difference in path length is calculated and shown to only depend on order, wavelength, and number of grooves illuminated. We report an experiment conducted to test these calculations. The temporal dispersion was measured for many gratings and spectral orders. Masks were used to limit the number of grooves illuminated as well as to study the relationship between the area of the grating illuminated and the temporal instrument function.

\section{II.THEORY}

Temporal broadening is introduced into our system by path length differences across the grating and the temporal resolution of the streak camera. Adding these errors in quadrature defines the error introduced by the spectrometer.

$$
\delta t_{s p}^{2}=\delta t_{g}^{2}+\delta t_{s c}^{2}
$$

The geometry in figure 1 explains how the grating causes the temporal smearing. The path difference of radiation reflected from adjacent groves is given by:

$$
\Delta l=d(\sin \alpha+\sin \beta)
$$

Each groove adds another $\Delta \mathrm{l}$ to the path difference. Summing over all grooves, the path difference for light rays reflected from opposite ends of the grating is:

$$
\begin{aligned}
\Delta l_{t o t} & =n d(\sin \alpha+\sin \beta) \\
& =G L d(\sin \alpha+\sin \beta) \\
& =G L m \lambda_{0}
\end{aligned}
$$

Photons arriving at the detector from opposite ends of the grating are thus offset temporally by : 


$$
\delta t_{g}=\frac{G L M \lambda_{0}}{c}
$$

From (4) it is clear that, for a given wavelength, the temporal dispersion depends directly on the grating, the order, and the number of grooves illuminated $\left(G^{*} L\right)$.

A more detailed discussion on the temporal resolution of a streak camera can be found elsewhere.

\section{EXPERIMENT}

To measure the time smear introduced by the grating, a $300 \mathrm{fs}$ laser pulse was propagated through a spectrometer and imaged by a streak camera with a temporal resolution of $\sim 1 \mathrm{ps}$. Since the laser pulse duration is much smaller than the cameras resolution, any variation or structure in the pulse would be un-resolvable and any measured temporal profile could be attributed to be primarily from optical path differences across the grating. Figure 2 shows a schematic of the spectrometer system used in the experiment. A $1 \omega$ laser pulse is frequency doubled and sent through the spectrometer. Before entering spectrometer, the $2 \omega$ light is focused in the plane of the entrance slit. The focusing lens is chosen so that the light passing through the entrance slit slightly overfills the spherical mirror, ensuring the grating is fully illuminated. Light reflected from the grating is focused by a second spherical mirror into the exit slit of the spectrometer and collected by the steak camera. The streak camera was operated at various sweep speeds from $0.5-5 \mathrm{~ns}$, depending on available laser signal. The temporal smearing was measured for $300,1200,1800,2400 \mathrm{l} / \mathrm{mm}$ gratings and for five spectral orders using the $300 \mathrm{l} / \mathrm{mm}$ grating. To test the dependence of the broadening on the number of grooves illuminated, measurements were made using a variety of optical masks. The masks were placed in front of the first spherical mirror to limit the area of the grating that is illuminated by the laser. Different mask geometries were used and correlated to the temporal instrument function.

\section{Results}

Figure 3 is a typical image recorded by the streak camera, in this case using a $180011 / \mathrm{mm}$ grating and a $1 \mathrm{~ns}$ sweep. The $300 \mathrm{fs}$ pulse has been stretched out by the spectrometer to 300 ps. Light imaged at different times can be though of as having been reflected from a different parts of the grating. Since the temporal pulse shape can't be resolved by the streak camera, the rise and fall of the signal is seen in the image as a sharp increase/decrease in intensity. These edges in the intensity provide a clear reference to the beginning and end of the signal, and thus a simple method for measuring of the temporal dispersion. A line out is taken over the region occupied by the pulse and a polynomial function is fitted to the background noise. Two lines are fitted to the sharp rise and fall of the signal and their intersections with the polynomial define the beginning and end of the pulse. The temporal dispersion is calculated from the measured width of the pulse. The lineout in figure $4 a$ shows a Gaussian like shape to the pulse. This shape is due to the spatial profile of the beam and is not related to the temporal instrument function of the spectrometer. Since the laser spot is imaged onto the grating, any variation in intensity along the length of the grating (orthogonal to grooves) will be detected as an intensity variation in time in the streak camera image. If an idealized pulse with a constant spatial profile were sent through the spectrometer, the corresponding temporal profile in the streak camera would also be constant, though stretched in time. A flatter spatial beam profile can be achieved by employing an optical mask on the spherical mirror. In this application, the mask serves to clip out the less intense wings of the beam, leaving behind a more even beam profile. As illustrated in figure $4 \mathrm{~b}$, the temporal profile introduced by the spectrometer also shows little variation in intensity. Another feature seen in the image is the temporal separation of wavelengths or "chirp," introduced by the finite bandwidth of the laser and the slight variations of incident angle across the grating. To understand the chirp it is important to realize that time the light arrives at the spectrometer is directly related to the position on the grating from which it was reflected. Light coming from 
the end of the grating closer to the camera arrives earlier than light from the grating's opposite end. Furthermore, light incident on different parts of the grating will make slightly different angles with respect to the grating normal. Corresponding to this variation in angles is a range of wavelengths that satisfy the grating equation for the given setup. Put another way, light from different sections of the grating constructively interferes at slightly different wavelengths. Thus the chirp can bee seen as a effect of light being reflected from different parts of the grating. It can be concluded that the chirp and time smearing are dependent on light coming from different parts of the grating. By masking the mirror, thus shortening the length of the grating illuminated, it is found that both the temporal and spectral smearing are reduced.

To determine the dependence of temporal broadening on grating, the experiment was run for $300,1200,1800,360011 / \mathrm{mm}$ gratings, using $.0 .5-2 \mathrm{~ns}$. As seen in figure 5 , the measured broadening fits the theory quite well. In a similar fashion, the dependence of the dispersion on spectral order was measured using the $300 \mathrm{ll} / \mathrm{mm}$ grating and a $0.5 \mathrm{~ns}$ sweep for $\mathrm{m}=1,2,3,4$ 5 . For higher spectral orders, the signal intensity was only slightly above the background, creating greater errors in the method for measuring the broadening. Since the signal is just above the noise, the sharp edges, introduce by the pulse, get distorted, creating more uncertainty in the lines fitted to the sharp edges. Again, the broadening measured from the data fits the theory quite well. To be able to vary the length of grid being illuminated, spectral masks were employed. By using rectangular, square, and diamond masks of varying sizes, it can be seen that the temporal broadening of the pulse depends on the length of the grating orthogonal to the grooves. Masking of the height of the grating only serves to lessen the intensity. Thus, using a $10 \mathrm{~cm} \times 5 \mathrm{~cm}$ mask will have the same temporal dispersion as a $5 \mathrm{~cm} \times 5 \mathrm{~cm}$ mask, but the signal from the latter will be significantly dimmer. The dependence of the dispersion of the length of grating illuminated was measured by using rectangular masks of different lengths (but same height). Since the mask was put in front of the mirror, the projection of the mask onto the grating must be used to determine the number of grooves illuminated. The broadening if found to be linear with respect to the mask length, as predicted by the theory.

\section{CONCLUSIONS}

The time smear introduced from path length through the detector has been analyzed and found to depend only on the order of the grating, wavelength, and the number of grooves illuminated, in accordance with the theory developed. Properties of the measure pulse, and effected introduced by optical masks were discussed and correlated to the temporal instrument function.

\section{ACKNOWLEDGMENT}

This work was performed under the auspices of the U.S. Department of Energy by Lawrence Livermore National Laboratory under Contract DE-AC52-07NA27344. This work was funded by the Laboratory Directed Research and Development Program at LLNL under project tracking code 06-ERD-056 
Figure1 ( PATH LENGTH GEOMETRY)

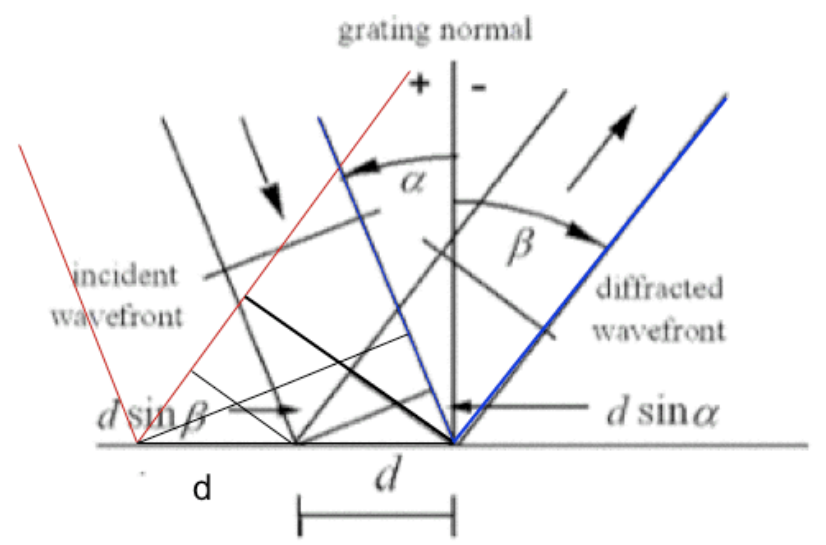

The path difference for the red beam and the blu beam is $2 d(\sin \alpha+\sin \beta)$

Figure2 (schematic of setup)

\section{Figure3}

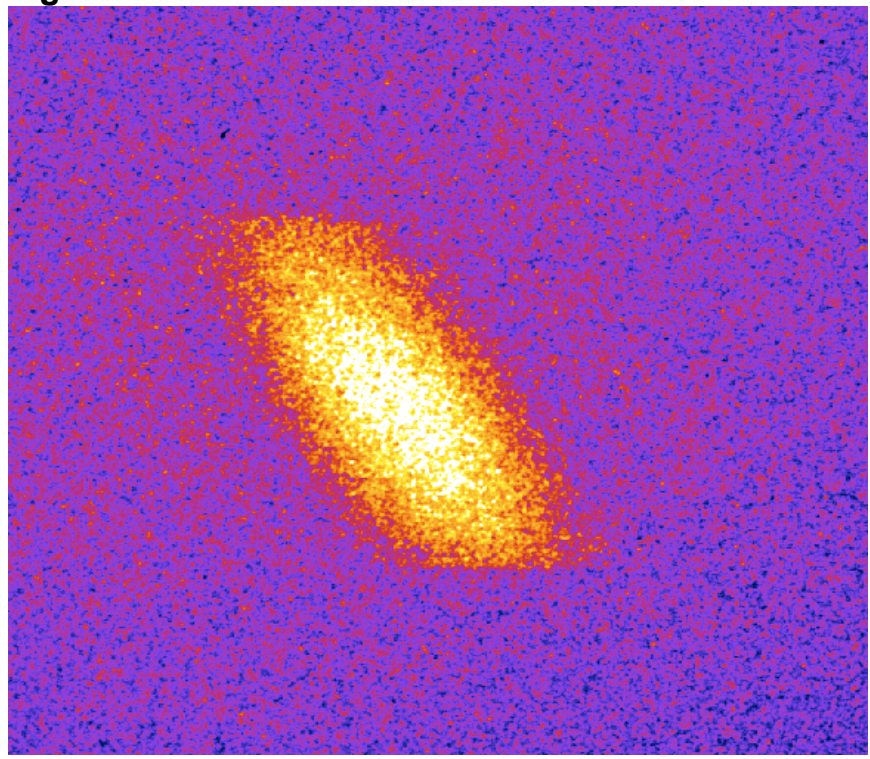

Figure 4a 


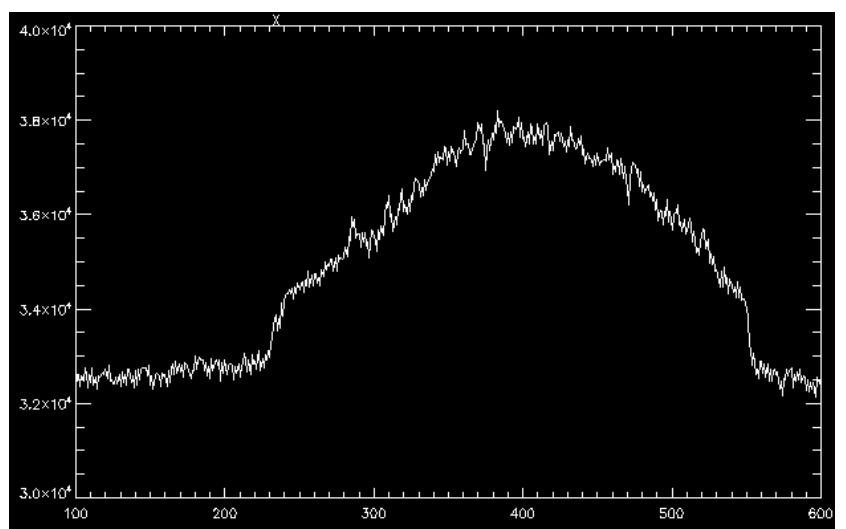

Figure $4 b$
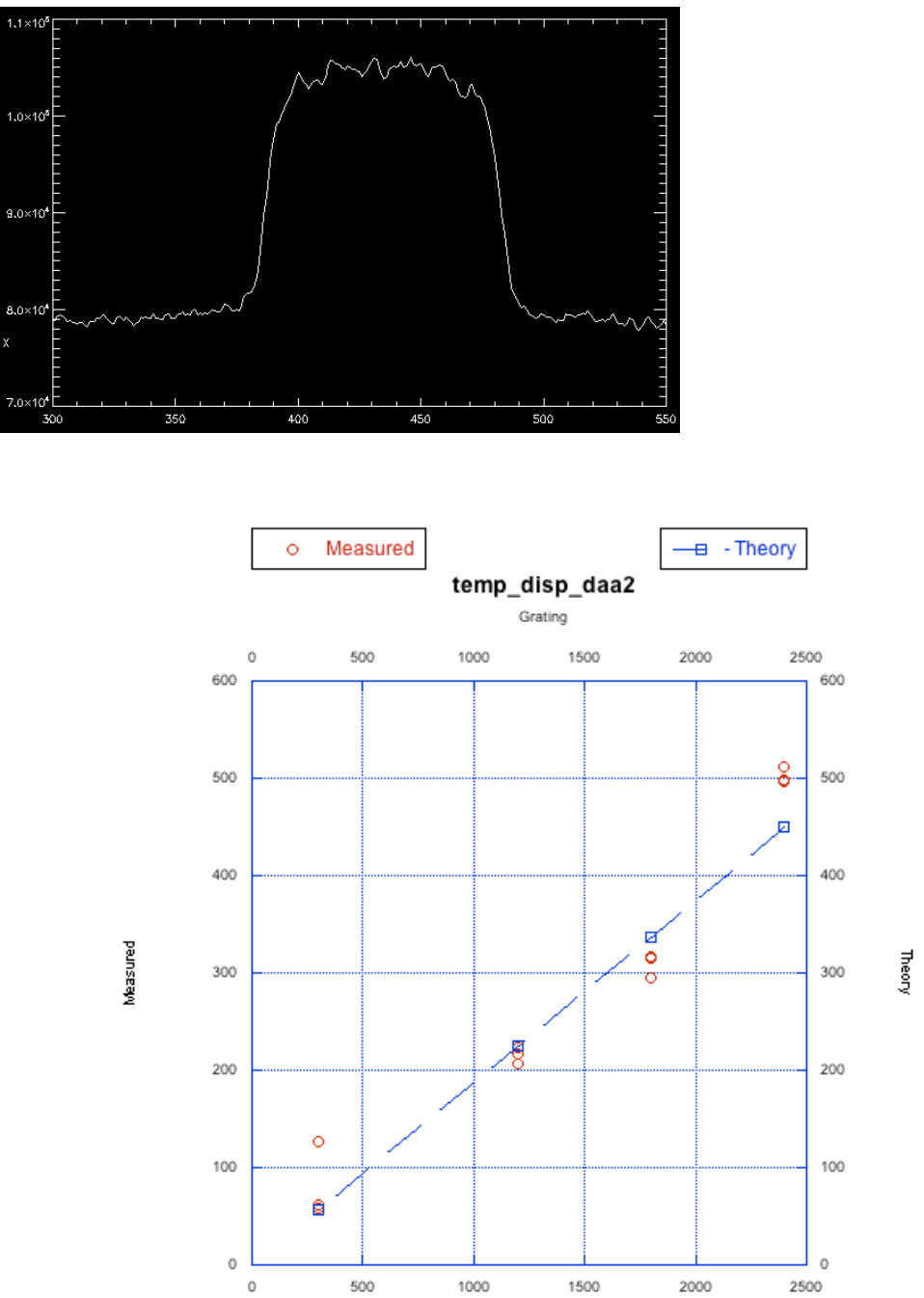

Figure5a

$$
\text { Grating }
$$




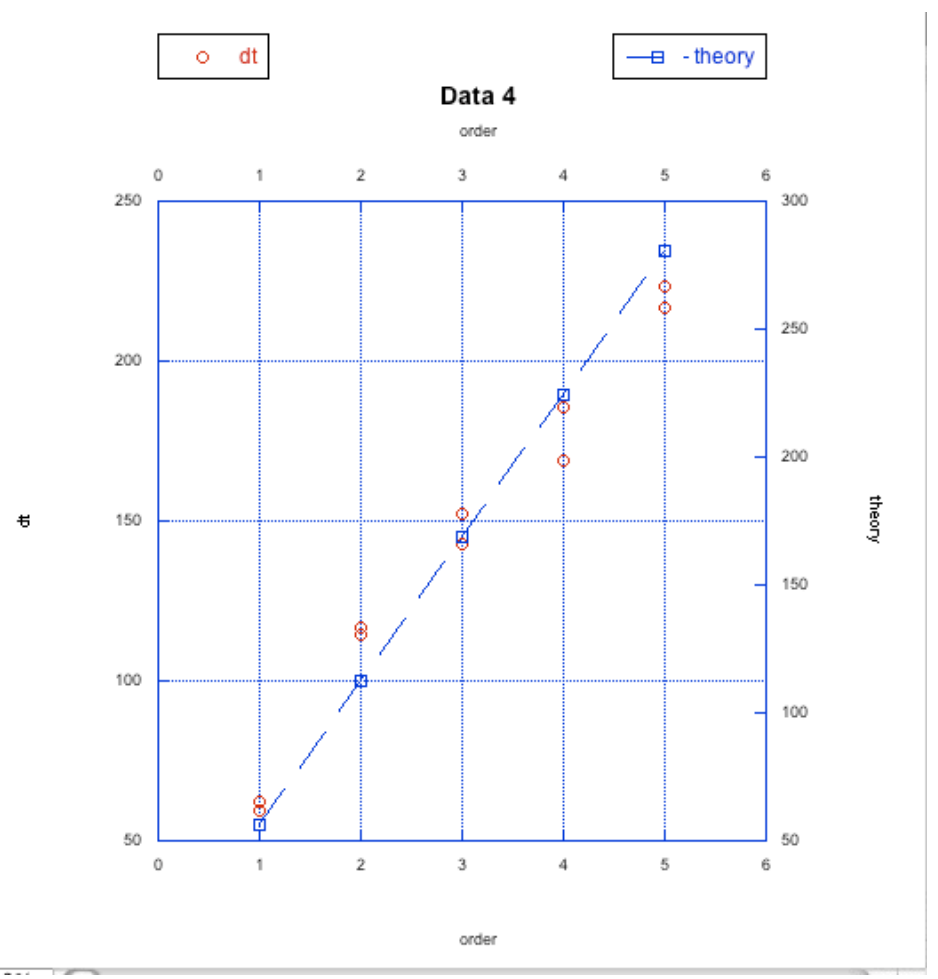

Figure $5 b$ 


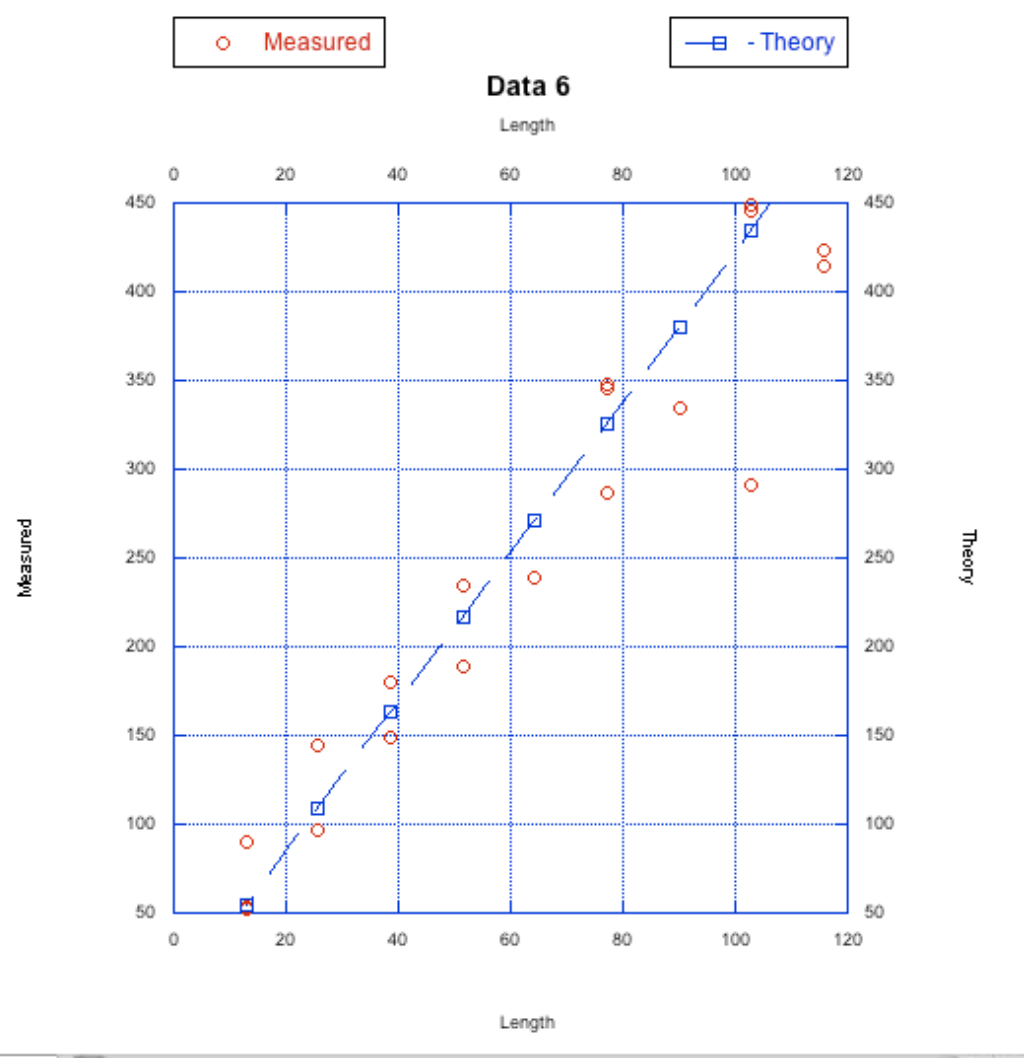

FIGURE 5C 